\title{
MOMENTI DI PRESENZA ITALIANA A COSTANTINOPOLI-ISTANBUL
}

\author{
Nevin Özkan \\ Università di Ankara (Turchia) \\ ozkann@ankara.edu.tr
}

\section{Riassunto}

La città fondata dall'Imperatore Costantino nel quarto secolo è ricca di presenza italiana dai tempi remoti fino a oggi. Questa presenza, avvertita non solo nel campo della storia, ma anche in quello dell'arte e della cultura, si rispecchia in diversi modi nella città sorta - come Roma - su sette colli. Volendo partire dal passato bizantino, si menzionerà la torre di Galata, costruzione che ancor'oggi domina la città bassa oltre il Corno d'oro e che conserva numerose memorie culturali e rimandi che rievocano l'Italia. Se si va al periodo della conquista di Costantinopoli, da parte di Fatih Mehmet (Maometto Secondo), è doveroso ricordare il quartiere di Pera, dove abitavano il bailo veneto e numerose famiglie di stessa provenienza. Pera fu nel Sei e Settecento la scena della formazione di una scuola di lingue per interpreti (secondo un modello ottomano già esistente), quindi si assistette a una concezione modernissima e importante di presenza di culture diverse. Verso il tardo Settecento e per tutto l'Ottocento Costantinopoli-Istanbul fu la meta di varie categorie di viaggiatori, tra cui missionari, uomini d'affari, turisti e scrittori come Gustave Flaubert, Pierre Loti, Edmondo de Amicis e nel Novecento Corrado Alvaro e Giuseppe Antonio Borgese, che ne hanno dato testimonianza scritta nelle loro opere. Inoltre, gli Italiani hanno lasciato la loro impronta indelebile attraverso le opere di architetti tra i quali Raimondo D’Aronco e pittori quali Fausto Zonaro, ammirati nella ex-capitale ottomana e stimati nella Turchia odierna.

Nella città di Costantinopoli-Istanbul si può tracciare, fin dal Medioevo, una presenza stabile di artisti italiani, che tutt'ora continua 
a persistere. L'attuale rappresentante è, senz'altro, il regista e scrittore Ferzan Özpetek, nato e cresciuto a Istanbul, che si definisce italiano e che costituisce un trait d'union tra le due culture con i suoi film, che non mancano di originalità e multiculturalità.

Parole chiave: Costantinopoli, Istanbul, Edmondo de Amicis, Corrado Alvaro, Fausto Zonaro, Raimondo D’Aronco, Galata, Özpetek 
Nella città fondata dall'Imperatore Costantino, che si trova all'estremità dell'Europa, a un chilometro o meno di distanza dall'Asia, seguiremo alcune tracce di presenza italiana a Istanbul. Testimonianze che continuano a essere importanti ancora oggi dal punto di vista storico, sociale, artistico e culturale.

Partendo dall'epoca medievale, si nota la torre di Galata, simbolo di Istanbul - una costruzione italiana di dimensioni enormi per l'epoca, che ancor oggi domina la parte bassa della città e il Corno d'oro. Se si va invece al periodo successivo, alla conquista dell'allora Costantinopoli da parte di Mehmet Fatih, si possono riconoscere nel quartiere di Pera numerosi rimandi alla cultura italiana. A Pera abitava il bailo veneto e abitavano altresì molte famiglie che lavoravano per l'impresa commerciale veneziana. Nel Seicento e nel Settecento, questo quartiere ospitò una scuola di lingua per interpreti. Le scuole di lingua del tipo di Berlitz e Linguaphone presentano un primissimo esempio di istituzioni volute dai veneziani, per educare i giovani prescelti alla conoscenza della lingua e della cultura ottomana, quindi a una concezione importante e modernissima di apertura a culture diverse. Alla fine, però, non sono stati i veneziani, ma gli ottomani a fondare per primi una scuola di lingue per interpreti (vedi Özkan, Speelman, 2013).

Verso il tardo Settecento, per tutto l'Ottocento e nel Novecento, Costantinopoli-Istanbul fu la meta di varie categorie di viaggiatori, fra cui missionari, businessmen, ma anche turisti e soprattutto scrittori. C’è un'enorme produzione letteraria di testi, non solo di famosi intellettuali francesi, ma anche di scrittori italiani, tra cui i celebri Edmondo De Amicis, Corrado Alvaro, Giuseppe Antonio Borgese e tanti altri, che hanno immortalato la 'seconda Roma' nelle loro opere. Riportiamo una descrizione di De Amicis di questa meravigliosa città (De Amicis, 1883, 17):

Mentre stavo guardando Scutari, il mio amico mi toccò col gomito per annunziarmi che aveva scoperto un'altra città. E vidi infatti, voltandomi verso il mar di Marmara, sulla stessa riva asiatica, al di là di Scutari, una lunghissima fila di case, di moschee e di giardini dinanzi a cui era passato il bastimento, e che fino allora erano rimasti nascosti dalla nebbia. Col cannocchiale si discernevano benissimo i caffè, i bazar, le case all'europea, gli scali, i muri di cinta degli orti, le barchette sparse 
lungo la riva. Era Kadi-Kioi, il villaggio dei giudici, posto sulle rovine dell'antica Calcedonia, già rivale di Bisanzio; quella Calcedonia fondata seicento ottantacinque anni prima di Cristo dai Megaresi, ai quali fu dato dall'oracolo di Delfo il soprannome di ciechi per avere scelto quel sito invece della riva opposta dove sorge Stambul. - E tre città - ci disse il Capitano -; e contino sulle dita perché a momenti ne salteranno fuori delle altre.

E ora leggiamo le parole di Corrado Alvaro (1995, 40):

La penisola su cui sorge la città non ha più l'aspetto d'una terra, ma di un regno profondo e cavo, echeggiante di cisterne, vòlte, archi, templi, acquedotti sotterranei, una città sepolta di cui si vede lo spaccato, su cui un'altra più tranquilla, più leggera, s'è piantata. Su questo regno sotterraneo di pietre, di marmi, di vòlte, si sono arrotondate, come bolle venute su da un mare abissale, le cupole dei bagni, dei seminari, delle moschee, volgono le spalle alla città, al mondo, a tutti, raccolte a triangolo intorno a cupola maggiore. La gente, come troppo stanca della lunga strada, costruì per sé baracche di legno là dove era la pietra della città antica, cui ne servivano anche per lapidare principi e sacerdoti; a ogni piccola avventura dei sette colli, sui gioghi aperti tra colle e colle, dove si respira il mare e la vicenda della strada, la stessa che seppe le processioni, i re coronati, le basilisse, gli usurpatori, furono adattate terrazze, loggette, chioschi, giardinetti, modesti palchetti per lo spettacolo della via minuto e chiassoso; fu tutta la fatica degli uomini, i quali vi stanno come in piccoli teatrini, con una fragilità che ricorda certe botteghe cinesi, esili, quasi per gioco.

Le citazioni sopra riportate costituiscono una testimonianza scritta dell'importanza che tutt'ora Costantinopoli-Istanbul riveste per gli Italiani. Infatti, questa città ha costantemente avuto una comunità italiana stabile, rintracciabile a partire dal Medioevo in poi, attraverso tutti i secoli successivi, anche se ci sono stati periodi in cui gli Italiani hanno preferito altri posti, soprattutto nel periodo in cui scoppiò la peste a Istanbul. Comunque, anche oggi si può parlare di una continua presenza di Italiani in città.

Prova importante della presenza italiana a Istanbul è anche quella del famoso orientalista Fausto Zonaro, che visse in un periodo in cui la pittura non era un genere d'arte molto diffuso in Turchia, non essendo prodotta nel Paese. Le opere di Zonaro incontrarono i favori della corte 
ottomana di Abdulhamid II e ornarono le pareti dei palazzi ottomani, in particolare quelle del palazzo di Dolmabahçe. Lo stesso Abdulhamid assunse come architetto prediletto un altro italiano, Raimondo d'Aronco (1857-1932 $)^{[1]}$, che lasciò in Oriente un bel numero di costruzioni molto interessanti in stile Art Nouveau. Nel 1893 l'architetto gemonese aveva fatto un primo viaggio in Turchia. L'anno successivo, ci fu un grande terremoto. D’Aronco divenne, per volontà del soldano Abdulhamid II, capo-architetto della ricostruzione della capitale di allora, cioè dell'odierna Istanbul. D’Aronco alternò soggiorni e progetti in Italia con soggiorni prolungati in Turchia, dove realizzò, tra l'altro, la residenza estiva dell'Ambasciata d'Italia a Therapia (1905).

La preferenza data a D’Aronco dovrebbe gettare una nuova luce anche sull'enigmatico soldano, considerato intrigante e reazionario, ma che compì grandi passi per portare il suo Impero ai livelli degli Stati europei. Dopo la deposizione del soldano, avvenuta nel 1909 a opera dei Jöntürkler, D’Aronco perse il posto di capo-architetto in Turchia e tornò definitivamente in Italia, dove realizzò, fra l'altro, il municipio di Udine, progettato ed eseguito però in uno stile diverso, più eclettico e forse meno interessante. D'Aronco influì talmente sull'architettura di Istanbul, che ancora oggi possiamo ammirare alcune delle sue opere nella ex-capitale ottomana.

Con il compositore di opere liriche e scultore Pietro Canonica (1869-1959; romano di adozione, ma nato in Piemonte) siamo intanto nel periodo repubblicano. Si tratta di un artista ancora oggi famoso, al quale il Comune di Roma ha dedicato un proprio museo a Villa Borghese, per ospitare il generoso lascito dell'artista. Il suo rapporto con la Turchia può sorprendere. Fu grazie alla geniale intuizione del padre della patria, Mustafa Kemal, che il giovane Stato in costruzione si erse a livello europeo,

[1] Raimondo d'Aronco fu un esponente del Liberty, quello stile che rinnovò l'architettura del periodo 1890-1915 rifiutando il passato e cercando il nuovo. Urbanisticamente, la sua fortuna è legata all'ingrandimento dei centri urbani nello stesso periodo in cui centinaia di migliaia di abitanti migrarono dalle campagne europee verso le città, che ebbero un lungo momento di esplosione demografica. Il fenomeno dilagò a Parigi, Praga, Riga, Vienna, Budapest e tante altre città e Istanbul non ne restò immune. I quartieri europei della metropoli ottomana, Galata e Pera e le loro estensioni verso Nord (Nişantaşı, Beşiktaş) ne videro i primi esempi, di solito creati da architetti formatisi in Europa, come alcuni armeni (cui è stata dedicata nel 2013 una bella mostra ad Ankara), qualche greco indigeno e l'italiano D'Aronco. Gli italiani allora costituivano a Istanbul una comunità fiorente di forse 30.000 mila abitanti, il che creava una distinta atmosfera italiana. 
ricorrendo alla recente arte del vecchio continente - arte necessaria a celebrare la rivoluzione nazionale nella persona del suo capo. Prima di Atatürk - come Kemal si chiamò nella campagna che diede a ciascun turco un cognome - nessun uomo era stato rappresentato in una statua, forma d'arte considerata contraria all'Islam, in quanto religione che respinge l'immagine pubblica di qualsiasi uomo. Canonica non solo seppe rappresentare il grande uomo in una posa che lo ritraesse allo stesso tempo come leader e come uomo (nella statua equestre a Ulus e nella statua sull'attuale Atatürk Bulvarı, entrambe situate ad Ankara), ma riuscì anche a dare un volto a coloro che lo avevano affiancato durante la guerra di liberazione. Forse il suo capolavoro è quello situato in piazza Taksim a Istanbul. In quest'opera lo sculture italiano ha saputo raffigurare Mustafa Kemal accanto al suo vice, Ismet Inönü e al maresciallo Fevzi Çakmak, come incarnazioni di una volontà popolare. Dietro a loro sono stati rappresentati, fra gli altri, Frunze e Vorošilov, in omaggio all'appoggio di Lenin alla Guerra d'Indipendenza. D'altronde, il monumento divenne un modello che avrebbe avuto una certa influenza su un tipo di scultura diffusasi poi nell'Unione Sovietica e che manca (che si sappia) nell'Italia mussoliniana e nella Germania nazista - regimes, infatti, che si rendevano conto di non rappresentare alcuna volontà popolare. Le statue di Canonica in Turchia (oltre ad Ankara e Istanbul, una sua statua equestre si trova pure nella terza grande città turca, Izmir, ovvero Smirne) riescono, invece, a suscitare sia fra gli artisti contemporanei (che le imitano) sia fra il popolo molto rispetto e ammirazione per un leader scomparso da quasi ottant'anni, ma vivo nel cuore della nazione.

Giulio Mongeri, architetto di grandi visioni e abilità, vissuto tra il 1873 ed il 1951, si differenzia dagli altri colleghi italiani, in quanto operò soprattutto nella nuova capitale, Ankara, definendone l'assetto urbanistico strutturale. Mongeri edificò palazzi di notevole importanza tutt'ora funzionanti, quali la sede della Banca del Lavoro Turco, la sede della Banca dell'Agricoltura e il Palazzo di Ankara, foresteria usata per ospitare illustri uomini di stato. Conclusi i lavori ad Ankara, Mongeri si diresse verso Bursa, grande e secolare città, famosa per il commercio della seta, ma anche per le sue acque termali, dove ideò e realizzò il grande albergo termale della città, Çelik Palas, ancora oggi in funzione. 
Diverso è il caso di Paolo Caccia Dominioni (1896-1992), un nobiluomo, conte e barone, d'antica stirpe milanese. A una vocazione artistica, che includeva il disegno e la letteratura, si affiancava in lui un sincero patriottismo. Combattè in ben quattro guerre: la Prima Guerra Mondiale (da volontario), la Guerra d'Africa, la Seconda Guerra Mondiale, oltremare e poi in Italia la Guerra partigiana. Militarmente parlando, fu quindi un vero eroe. Il progetto per un'ambasciata italiana ad Ankara fu tra le sue opere più riuscite. Se si volesse collocare Caccia Dominioni in una determinata corrente, sarebbe forse quella della "neue Sachlichkeit" (da tradurre con "funzionalismo") degli anni '30 dello scorso secolo, che rientrava anche nella gamma di stili voluta dal regime. Se Caccia Dominioni adottò questo stile, non fu senza l'eleganza tipica italiana e l'inquadramento nel paesaggio da lui voluto anticipò una forma di landscape art poi teorizzata da Bruno Zevi come "architettura organica". L’Ambasciata, progettata e realizzata da Caccia Dominioni, offre ancora oggi un bell'esempio della creatività dellarchitetto italiano, che pensò alla funzionalità del suddetto complesso articolato in tre palazzi e una chiesa.

In terra turca approdarono anche musicisti italiani, tra i quali ricorderemo Giuseppe Donizetti (1788-1856) e Callisto Guatelli (18191899), che eseguirono con molti apprezzamenti la loro arte nell'Impero Ottomano dell'Ottocento.

Parlando di presenza italiana sul suolo turco, non è da dimenticare la Società Operaia di Mutuo Soccorso, fondata nel 1863 a Istanbul, la quale ospitò tra il 1828 e il 1831 Giuseppe Garibaldi, che ne fu anche il presidente. La Società è oggi ancora operativa, seppur con un numero di membri assai ridotto.

Tra le altre opere realizzate in Turchia da Italiani vanno ricordate: l'Istituto Italiano di Cultura, varie associazioni culturali, il Circolo di Roma, testate giornalistiche come "La Rassegna Italiana" (pubblicata dalla Camera di Commercio Italiana di Istanbul), "La Gazzetta di Istanbul" (il bollettino mensile del Circolo di Roma), diverse banche e ditte di costruzione, che hanno realizzato grandi progetti nella parte centrale e a est del Paese, come la diga di Gokçekaya, quella di Karakaya e il tunnel che collega Istanbul con l'Anatolia. Sono tutti importanti progetti, attraverso i quali l'Italia è riuscita a garantire una distinta presenza, un "marchio", anche nella Turchia attuale. 
Venendo al giorno d'oggi, forse ancora più importante e interessante è la presenza del regista Ferzan Özpetek, che costituisce una specie di trait-d'union tra le due culture: quella turca e quella italiana. Anni fa Özpetek ha, infatti, cambiato la grafia del suo nome in Ozpetek, definendosi più italiano che turco. Dopo il suo primo film Hamam, la cui trama si svolge a Istanbul e un secondo film, Harem Suare, girato nella stessa città, ha scelto successivamente l'Italia come ambientazione per i suoi lavori. Dobbiamo aggiungere che Özpetek ha ottenuto ultimamente un notevole successo anche come scrittore. I suoi libri Rosso Istanbul - da cui è stato tratto un omonimo film e Sei la mia vita sono stati tradotti in molte lingue e attirano l'attenzione di appassionati per la loro originalità e la loro dimensione multiculturale.

Attraverso le manifestazioni artistiche sopraelencate, gli Italiani hanno da sempre testimoniato la loro presenza a Costantinopoli-Istanbul, lasciando tracce indelebili. Ancora oggi ci sono a Istanbul un quartiere italiano, un ospedale italiano e tre scuole italiane. Inoltre, è in fase di costruzione un'università italo-turca; iniziativa che spiega molto bene la rilevanza delle connessioni culturali tra queste due civiltà; importanza riconosciuta non solo dagli Italiani, ma anche dai Turchi.

Forse il punto in comune di tutti gli artisti, musicisti e architetti italiani che hanno lavorato a Istanbul è che hanno mirato a creare opere d'arte non solo esteticamente belle, ma opere in cui si fondono elegantemente e artisticamente elementi orientali con quelli italiani. Il loro successo è confermato dal fatto che ancora oggi esse ci affascinano e ci ispirano; spronandoci a lavorare nel nostro campo - qualunque esso sia - con un approccio interculturale, che va oltre i confini meramente geografici, seguendo la via indicata dal grande Atatürk. 


\section{BIBLIOGRAFIA}

ALVARO, C., 1995, Viaggio in Turchia, Monteleone, Vibo Valentia.

ÇINICI, D., 2015, Giulio Mongeri, an Architect Efficient in the Building of Capital City Ankara and his Biography, in "Ankara Araştırmaları Dergisi", 3(1), 13-41.

DE AMICIS, E., 1883, Costantinopoli, Fratelli Treves, Milano.

EYICE, S., 1986, Atatürk ve Pietro Canonica, Eren, İstanbul.

GRAZIANO, V. (a cura di), 1994, Ambasciate d'Italia in Turchia, Mediterraneum, Catania.

Istituto Italiano di Cultura di Istanbul (a cura di), 1995, Raimondo D’Aronco in Turchia (1893-1909), Progetti della Galleria di Arte Moderna di Udine, Guzel Sanatlar Matbaasi A. S., Istanbul.

ÖZKAN, N., SPEELMAN, R., 2013, I dragomanni/Dragomanlar, in Pedani, M.P. (a cura di), Il Palazzo di Venezia a Istanbul e i suoi antichi abitanti, Edizioni Ca’ Foscari - Digital Publishing, Venezia. 


\section{THE MOMENTS OF ITALIAN PRESENCE IN CONSTANTINOPLE - ISTANBUL}

The city founded by the Emperor Constantine has been full of signs of Latin presence over past centuries until today. These signs are reflected not only in the historical, but also in the artistic and cultural fields in the city situated on seven hills. One could start by naming the Galata tower that even today dominates the lower city beyond the Golden Horn, conserving many cultural memories and associations, which bring Italy to mind. If one is to go back to the time of the conquest of Constantinople - Istanbul by Fatih Mehmet, it is a must to remember the district of Pera where not only the Venetian bailo, but also many Venetian families used to live. Pera was in fact the place where a school of translators was founded in the seventeenth and eighteenth centuries so we witness quite a modern and different conception of culture. Towards the end of the eighteenth and throughout the nineteenth and twentieth century Constantinople - Istanbul became the destination of various categories of travelers; missionaries, businessmen, tourists and writers, such as Gustave Flaubert, Pierre Loti, Edmondo de Amicis, Corrado Alvaro and Giuseppe Antonio Borgese, who in their works gave an account of the city as it used to be. Moreover, the Italians have rendered themselves immortal in the City thanks to the works of architects such as Raimondo d'Aronco and painters such as Fausto Zonaro, admired in the Ottoman ex-capital and highly esteemed in a nowadays Turkey. In fact, it is possible to find traces of Italian presence in the City dating back to the Middle Ages still present with a hospital, churches, schools, associations and newspapers. The most actual and significant representative of Italy in Turkey is, without doubt, a film director and writer Ferzan Özpetek. He was born and raised in Istanbul and defines himself as an Italian thus forming a trait d'union between the two cultures with his original and multicultural films.

Key words: Costantinople, Istanbul, Edmondo de Amicis, Corrado Alvaro, Fausto Zonaro, Raimondo D’Aronco, Galata, Özpetek 


\section{TALIJANSKA PRISUTNOST U CARIGRADU — ISTANBULU}

Grad kojega je utemeljio car Konstantin u 4. stoljeću već mnogo stoljeća obiluje znakovima latinske prisutnosti. Ta se prisutnost odražava ne samo u povijesnim, već i u umjetničkim i kulturnim područjima grada koji se - poput Rima - nalazi na sedam brežuljaka. Izuzevši bizantsku povijest, moglo bi se započeti imenovanjem tornja Galata koji danas vlada nižim dijelom grada izvan Zlatnoga roga, koji čuva mnoge kulturne uspomene i udruge koje prizivaju Italiju. Ako se treba vratiti u vrijeme osvajanja Carigrada - Istanbula od strane Fatiha Mehmeta, moramo spomenuti kvart Pere gdje su živjeli venecijanski bailo i mnoge venecijanske obitelji. Pera je, zapravo, u sedamnaestome i osamnaestome stoljeću bilo mjesto osnivanja škole prevoditelja, pa svjedočimo modernoj koncepciji posve drugačije kulture. Krajem osamnaestoga i tijekom devetnaestoga i dvadesetog stoljeća Carigrad - Istanbul je postao destinacijom raznih kategorija putnika, poput misionara, poslovnih ljudi, turista i pisaca, kao što su Gustave Flaubert, Pierre Loti, Edmondo de Amicis, Corrado Alvaro i Giuseppe Antonio Borgese, koji su o gradu svjedočili u svojim djelima. Štoviše, Talijani su postali vječiti u gradu zahvaljujući radovima arhitekata, kao što su Raimondo d'Aronco i slikara poput Fausta Zonara, cijenjeni u osmanskome bivšem glavnom gradu i u današnjoj Turskoj. Zapravo je još iz srednjega vijeka u Carigradu - Istanbulu moguće pronaći tragove talijanske prisutnosti koja je i danas nazočna zahvaljujući bolnici, crkvama, školama, udrugama i talijanskim novinama. Najznačajniji predstavnik Italije u Turskoj je, bez sumnje, redatelj i pisac Ferzan Özpetek. Rođen je i odrastao u Istanbulu, izjašnjava se Talijanom te svojim originalnim i višekulturalnim filmovima predstavlja trait d'union dviju kultura.

Ključne riječi: Carigrad, Istanbul, Edmondo de Amicis, Corrado Alvaro, Fausto Zonaro, Raimondo D’Aronco, Galata, Özpetek 
